MONOGRÁFICO: Materiales, transporte y producción. Pósters del Workshop Internacional de Arqueología de la Construcción V, (Universidad de Oxford, 11-12 Abril 2015 / MONOGRAPH: Materials, transport and production. Posters of the 5th International Workshop on the Archaeology of Roman Construction, (University of Oxford, April 11-12, 2015)

\title{
Ubicazione delle cave di pietra da calce utilizzata come materia prima degli intonaci romani nella Lombardia occidentale
}

\section{Location of quarries of magnesian lime used as raw material of Roman plasters in western Lombardy (Italy)}

\author{
Roberto Bugini e Luisa Folli \\ CNR - Istituto per la Conservazione e la Valorizzazione dei Beni Culturali \\ e-mail: bugini@icvbc.cnr.it; lufolli@gmail.com
}

\begin{abstract}
RIASSUNTO
La calce come materiale da costruzione ebbe larga diffusione nell'architettura romana della Lombardia occidentale. La presenza di carbonato di magnesio è stata per lo più riscontrata negli intonaci dipinti provenienti dai siti romani di Milano. Il carbonato di magnesio testimonia l'impiego di dolomia per la preparazione della calce e rocce di questa composizione affiorano per esteso nelle Prealpi lombarde: dolomie e calcari dolomitici grigio chiari (Dolomia del Salvatore, Ladinico-Anisico), dolomie grigie, talvolta con ciclotemi (Dolomia principale, Norico). Non sono conservate evidenze di cave o di forni romani in quest'area prealpina: i Romani sfruttarono gli stessi affioramenti dolomitici, situati lungo la sponda orientale del lago Maggiore, affioramenti da cui si produsse calce a partire dal Medio Evo in avanti.

I depositi fluvio-glaciali del medio corso del fiume Adda tra Brivio e Trezzo (massi, ciottoli di calcari marnosi, calcari e dolomie che affiorano nel bacino fluviale) furono un'altra fonte medievale e moderna di materia prima, soprattutto per produrre una calce debolmente idraulica (chiamata "calce forte"), ma questo tipo di calce è assente negli intonaci romani.
\end{abstract}

Parole chiave: calce; calce magnesiaca; dolomia; intonaco romano; Lombardia.

\begin{abstract}
Lime as building material was widely diffused in Roman architecture of western Lombardy. The presence of magnesite (magnesium carbonate) was detected in painted plasters coming from roman sites of Milano: magnesite indicates the use of dolomite to make the lime. Dolomite rocks widely outcrop in the Lombard Prealps: light grey dolomites and dolomitic limestones (Dolomia del Salvatore, Ladinian-Anisian); grey dolomites, sometimes with cyclothemes (Dolomia Principale, Norian). There is no evidence of Roman lime quarries or kilns in this area; the Romans probably exploited the same dolomite outcrops, located along the eastern shore of lake Maggiore, where lime was produced from the Middle Ages onwards. The glacio-fluvial deposits of the middle course of the river Adda (cobbles, pebbles of siliceous limestone together with limestone and dolomite outcropping in the river basin) were another medieval and modern source of raw material, mainly to make a moderately hydraulic lime (called "calce forte"), but this kind of lime is lacking in Roman plasters.
\end{abstract}

Keywords: lime; magnesian lime; dolomite; Roman plaster; Lombardy.

Recibido: 09-06-2016. Aceptado: 12-07-2016.

Cómo citar este artículo / Citation

Bugini, R. y Folli, L. 2016: "Ubicazione delle cave di pietra da calce utilizzata come materia prima degli intonaci romani nella Lombardia occidentale", Arqueología de la Arquitectura, 13: e049. doi: http://dx.doi.org/10.3989/arq.arqt.2016.167 


\section{INTRODUZIONE}

La calce è uno dei più antichi e più importanti materiali da costruzione e costituisce un componente, definito legante dal punto di vista tecnologico che, miscelato con l'aggregato e l'acqua in proporzione variabile, serve a produrre le malte per gli intonaci, per gli stucchi, per le fondazioni di pavimenti a mosaico o ad opus sectile, per l'allettamento delle murature in ciottoli, in pietra da taglio o in laterizio.

La calce ha avuto un larghissimo impiego nell'architettura romana della città di Milano e della Lombardia occidentale, ma in assenza di testimonianze dirette, quali cave o fornaci, si è ritenuto valido procedere per via analitica. L'obiettivo è l'identificazione delle fasi mineralogiche del legante usato negli intonaci provenienti dai siti archeologici, per individuare quale fosse la materia prima utilizzata in quel periodo e la sua provenienza.

La metodologia adottata per l'indagine è la diffrattometria ai raggi $\mathrm{X}$ (XRD), su campioni ridotti in polvere.

\section{LINEAMENTI GEOLOGICO- PETROGRAFICI DELLA LOMBARDIA}

La regione Lombardia si estende su un vasto territorio compreso fra le Alpi (4049 metri di altezza al Pizzo Bernina) e la pianura alluvionale, a pochi metri sul livello del mare; la distanza media tra lo spartiacque alpino ed il corso del fiume Po è di 140 chilometri.

Il territorio può essere geologicamente suddiviso, da Nord a Sud, in tre fasce parallele. La prima coincide con la catena Alpina; dal punto di vista geologico si incontrano il Basamento scistoso comprendente rocce metamorfiche come gneiss, micascisti e marmi (sottoforma di lenti intercalate negli gneiss e nei micascisti), alcuni plutoni magmatici con rocce di tipo granitico (plutoni dei Laghi) o di tipo granodioritico e dioritico (plutone della val Màsino e plutone dell'Adamello) e affioramenti di rocce vulcaniche (porfidi e porfiriti del Varesotto e della Valle Camonica). La seconda fascia coincide con le Prealpi, denominate Alpi Calcaree Meridionali dal punto di vista geologico, comprendenti rocce carbonatiche del Mesozoico (calcari, calcari marnosi, dolomie), rocce detritiche del Terziario (arenarie e conglomerati) e del Quaternario (conglomerati); inoltre sono presenti depositi glaciali con massi erratici di natura magmatica o metamorfica provenienti dall'area alpina. La terza fascia coincide con la pianura Padana, ovvero la pianura alluvionale del Po e dei suoi affluenti; geologicamente si tratta di depositi sciolti di natura morenica e fluviale (Quaternario e attuale) a diversa granulometria (blocchi, ciottoli, ghiaie, sabbie, argille).

$\mathrm{Da}$ questo territorio provengono rocce che hanno fornito e che forniscono tuttora materiali da costruzione (blocchi grezzi, conci lavorati, pietre da taglio, lastre ecc.), alcuni dei quali utilizzati ininterrottamente fin dall'epoca romana: graniti di Baveno e di Montorfano, Ghiandone e Serizzo della Val Màsino, Tonalite dell'Adamello, Granofiro di Cuasso e Porfido di Bienno per quanto riguarda le Alpi; calcare nero dei laghi di Como e d'Iseo, dolomia di Angera, calcare di Viggiù e di Saltrio, calcare "Majolica", arenaria di Sàrnico, Ceppo del Brembo, calcare dolomitico di Botticino (Prealpi), senza trascurare i marmi e le diverse pietre colorate (marmo di Candoglia, marmo di Musso, Macchiavecchia e Rosso di Arzo, Arabescato Orobico della val Brembana, alabastri della Val Seriana, Occhiadino della val Camonica ecc.) che tanta parte ebbero nell'architettura e nella decorazione del Barocco e del Rococò. I deposti sciolti sono stati cavati negli anfiteatri morenici oppure negli alvei dei fiumi ed hanno fornito blocchi e ciottoli per le murature, ghiaie e sabbie per gli aggregati delle malte e argille per la fabbricazione dei laterizi.

\section{LA PRODUZIONE DI CALCE IN EPOCA STORICA}

Una considerazione specifica riguarda la calce, cioè la materia prima del legante delle malte, ottenuta dalla cottura di rocce sedimentarie carbonatiche.

Sulla base dei lineamenti geologici, le formazioni calcaree e dolomitiche, adatte alla preparazione della calce, si trovano nella fascia prealpina. Lo sfruttamento ha interessato soprattutto le formazioni di natura dolomitica rispetto a quelle di natura calcarea. Sono infatti ben documentate le fornaci che utilizzavano, fino al 1950, gli affioramenti di roccia dolomitica del Triassico ubicati in diversi siti della sponda orientale del lago Maggiore da Angera a Luino ed in aree limitrofe $(\mathrm{Cu}-$ rioni 1877; Fieni 2000).

All'ambito prealpino appartengono anche altri importanti siti di approvvigionamento: i depositi sciolti quaternari di tipo fluvio-glaciale del medio corso dell'Adda (Breislak 1822; Curioni 1877), depositi sfruttati fino all'inizio del Novecento. 


\section{LE CITAZIONI DEGLI AUTORI ANTICHI}

Sono stati considerati diversi trattati riguardanti l'architettura e le costruzioni in genere, pubblicati sia in epoca antica che in epoca medievale e moderna: sono rari quelli che riportano dettagli riguardo alla preparazione della calce.

Vincenzo Scamozzi, nel suo trattato incompiuto, fornisce una precisa testimonianza riguardo all'Italia settentrionale ed alla Lombardia. I principali siti sfruttati al principio del Seicento sono gli affioramenti dolomitici del lago Maggiore: “(...) le pietre ottime e migliori delle altre per far calcine sono (...) le pietre bianche di color vivo (...) e che hanno una grana marmorina come specialmente quelle del Ticino e di altri fiumi intorno al lago Maggiore (...)". Inoltre, Scamozzi descrive i depositi sciolti del medio corso dell'Adda e di altri fiumi limitrofi: "In queste nostre parti dall'uscita delle valli per 12 e 15 miglia nella pianura (...) si ritrovano le pietre da far calcina (...) e molto più lungo l'Adda nel Ducato di Milano, (...)" (Scamozzi: parte 2, libro 7, 17).

In precedenza, alcuni Autori latini, come Plinio (Plinius: libro 36, 174) e Palladio (Palladius: libro 1, 10,3), descrissero la calce ottenuta sottoponendo a cottura sia le pietre cavate da un affioramento roccioso sia i ciottoli fluviali sciolti; in questo secondo caso si sceglievano quelli di colore chiaro, riferibili secondo le attuali conoscenze alle rocce carbonatiche, e non quelli di colore scuro, riferibili alle rocce silicatiche. I due diversi tipi di approvvigionamento di pietra da calce sono descritti anche da Leon Battista Alberti (Alberti: libro
2, 11), da Andrea Palladio (Palladio: libro 1, 5) e dai traduttori del De Architectura di Vitruvio, come Daniele Barbaro (Barbaro: libro 2, 5).

\section{LE INDAGINI NEI SITI ARCHEOLOGICI}

Sono stati esaminati campioni di malte romane provenienti da diversi siti archeologici milanesi (Bugini e Folli 2013). Le indagini scientifiche riguardano, in maniera quasi esclusiva, le malte per intonaco, mentre altre tipologie sono rappresentate in maniera trascurabile: questa scelta è stata dettata dalle esigenze della Soprintendenza Archeologica della Lombardia, responsabile dei siti e committente delle indagini stesse.

Gli intonaci presentano di norma due strati sovrapposti: in quello inferiore alla calce è associato un aggregato composto da sabbie di natura quarzoso-silicatica; in quello superiore alla calce è associato un aggregato di natura carbonatica in genere frantumato artificialmente (cristalli di calcite, frammenti di rocce calcaree e/o dolomitiche). Le indagini diffrattometriche hanno messo in risalto, in entrambi gli strati, un dato particolarmente significativo: la presenza di magnesite nel legante. La magnesite (carbonato di magnesio), registrata alla scheda 8-479 dell'International Centre for Diffraction Data di Newtown Square (Philadelphia, Pennsylvania), testimonia con precisione l'uso di rocce dolomitiche per la preparazione della calce.

La tabella riporta i siti, la datazione, il numero dei campioni esaminati (Fig. 1).

\begin{tabular}{|l|l|c|c|}
\hline \multicolumn{1}{|c|}{ Sito } & \multicolumn{1}{c|}{ Datazione } & Campioni & Composiz. legante \\
\hline via Broletto & I secolo d.C. & 8 & calcite, magnesite \\
\hline via Correnti & I-III secolo d.C. & 9 & calcite, magnesite \\
\hline via Correnti & II secolo d.C. & 25 & calcite, magnesite \\
\hline piazza Fontana & metà I secolo d.C. & 6 & calcite, magnesite \\
\hline piazza Fontana & inizio I secolo d.C. & 2 & calcite, magnesite \\
\hline via Illica & III secolo d.C. & 6 & calcite, magnesite \\
\hline piazza Meda & metà I secolo d.C. & 1 & calcite, magnesite \\
\hline piazza Meda & inizio IV sec. d.C. & 6 & calcite, magnesite \\
\hline Università Cattolica & I secolo d.C. & 17 & calcite, magnesite \\
\hline Università Cattolica & III secolo d.C. & & \\
\hline
\end{tabular}

Fig. 1. Tabella - Siti archeologici di Milano - Malte per intonaco 


\section{LA CALCE MAGNESIACA}

La magnesite è il risultato della carbonatazione dell' $\mathrm{i}$ drossido di magnesio (brucite) presente in una calce spenta ottenuta dalla cottura di una dolomia. La dolomia, composta da carbonato doppio di magnesio e calcio, se riscaldata ad una temperatura di circa $900^{\circ} \mathrm{C}$ produce ossido di magnesio (periclasio) ed ossido di calcio (calce viva), liberando biossido di carbonio. I successivi gradi del processo, l'idratazione e la carbonatazione, comportano quindi la formazione dei rispettivi idrossidi (brucite e portlandite) e in seguito (presa e indurimento) quella dei carbonati (magnesite e calcite).

Non sussistendo evidenze di cave o di fornaci per calce di epoca romana nel territorio lombardo, si ritiene che la calce magnesiaca utilizzata in quell'epoca provenga necessariamente dalla cottura di dolomie. Rocce di questo tipo affiorano, nella Lombardia occidentale, in alcune aree dove sono presenti cave e formaci medievali e moderne: si può quindi presumere che le cave di epoca romana fossero aperte negli stessi luoghi, sfruttando probabilmente anche i materiali sciolti.

Questa affermazione si basa su quanto osservato riguardo alle pietre da costruzione utilizzate nella Lombardia romana (Bugini e Folli 2014). E' stato infatti rilevato come nei siti di cava sfruttati in epoca romana, per ottenere pietre da costruzione (ad esempio la dolomia di Angera, il marmo di Musso, i massi erratici della Brianza, il conglomerato del Brembo, il calcare dolomitico di Botticino ecc.), la coltivazione sia proseguita anche nei secoli successivi, giungendo talvolta fino i nostri giorni. E' lecito quindi supporre che, anche nelle cave di pietra da calce sfruttate in epoca romana, la coltivazione sia poi proseguita nel Medio Evo e nei secoli successivi.

L'uso di calce magnesiaca ottenuta dalla cottura di dolomie è documentato nell'area lombarda dal XII al XIX secolo (Bugini, Folli e Lommano 1996) in edifici dell'area genovese (Montagni 1988) e in edifici medievali del Tirolo (Austria e Italia) (Diekamp, Konzett, Wertl, Tessadri e Mirwald 2008). All'estero si segnala l'impiego di calci magnesiache in Inghilterra fino al XIX secolo (Seeley 2000) e negli Stati Uniti centro-orientali dal XVIII secolo (Schork 2012). In Lombardia erano interessate le citate dolomie del lago Maggiore, a Genova la "Dolomia principale" nell'area di monte Gazzo presso Sestri Ponente, nel Tirolo le dolomie triassiche dell'area del Brennero; in Inghilterra quelle della Permian Belt (da Durham a Nottingham), negli
Stati Uniti le dolomie paleozoiche dell'Illinois e della Pennsylvania (the Appalachians).

L'individuazione delle proprietà della calce magnesiaca non rientra nello scopo di questo studio. E' tuttavia interessante annotare i pareri discordanti della trattatistica ottocentesca. Secondo Curioni la calce ottenuta dalla cottura delle dolomie "ha la proprietà di abbandonare, più facilmente di molte altre pietre da calce, l'acido carbonico e di fornire calci dette grasse, che si rigonfiano molto coll'idratarsi, e a peso uguale forniscono per ciò una maggiore quantità di grassello (...)" e la dolomia è stata, in Lombardia, "la pietra da calce più in uso da tempi immemorabili" (Curioni: parte 2, capitolo 5). Secondo Cavalieri San Bertolo, invece, "Le pietre che contengono dell'allumina e della magnesia, sono le meno adatte a produrre la buona calcina" (Cavalieri San Bertolo: libro 3 , sezione 1, capitolo 4). Questa opinione è convalidata anche nei testi francesi ("calcari mescolati con forti proporzioni di magnesia producono le calci magre non idrauliche" - Jagnaux 1885). Studi successivi mettono, invece, in evidenza la migliore lavorabilità (Mills 1915) e le migliori caratteristiche meccaniche (Atzeni, Massidda e Sanna 1996) e di durabilità (Klemm 1989) riscontrabili nelle calci magnesiache in confronto con le calci calciche.

\section{LA CALCE DEL LAGO MAGgIORE}

La sponda orientale del lago Maggiore è interessata, sia nella parte settentrionale sia in quella meridionale, da modesti affioramenti di dolomie del Triassico adatti per la produzione della calce e ubicati in piccoli rilievi collinari, tuttavia ben evidenti nella geomorfologia della riva lacustre (Fig. 2). Le rocce interessate sono: dolomie grigiastre e calcari dolomitici (formazione geologica "Dolomia del Salvatore", Norico) tra Brezzo di Bedero e Caldè (parte settentrionale, Fig. 3, 4); dolomie grigie sovente con ciclotemi (formazione geologica "Dolomia Principale", Ladinico-Anisico) a Ispra e ad Angera (parte meridionale, Fig. 5). Altri affioramenti, anch'essi oggetto di sfruttamento almeno a partire dal XVII secolo, sono ubicati a Rasa e ad Arcisate ("Dolomia del Salvatore") oppure a Brenno Useria ("Dolomia Principale"); in questi casi, la distanza dalla sponda orientale del lago Maggiore, quindi dalla via d'acqua adatta al trasporto, è superiore a venti chilometri (Curioni 1877; Fieni 2000).

Indagini specifiche, condotte mediante spettrofotometria per fluorescenza ai raggi X (XRF) su calci preparate a partire dalle dolomie cavate sul lago Maggiore 


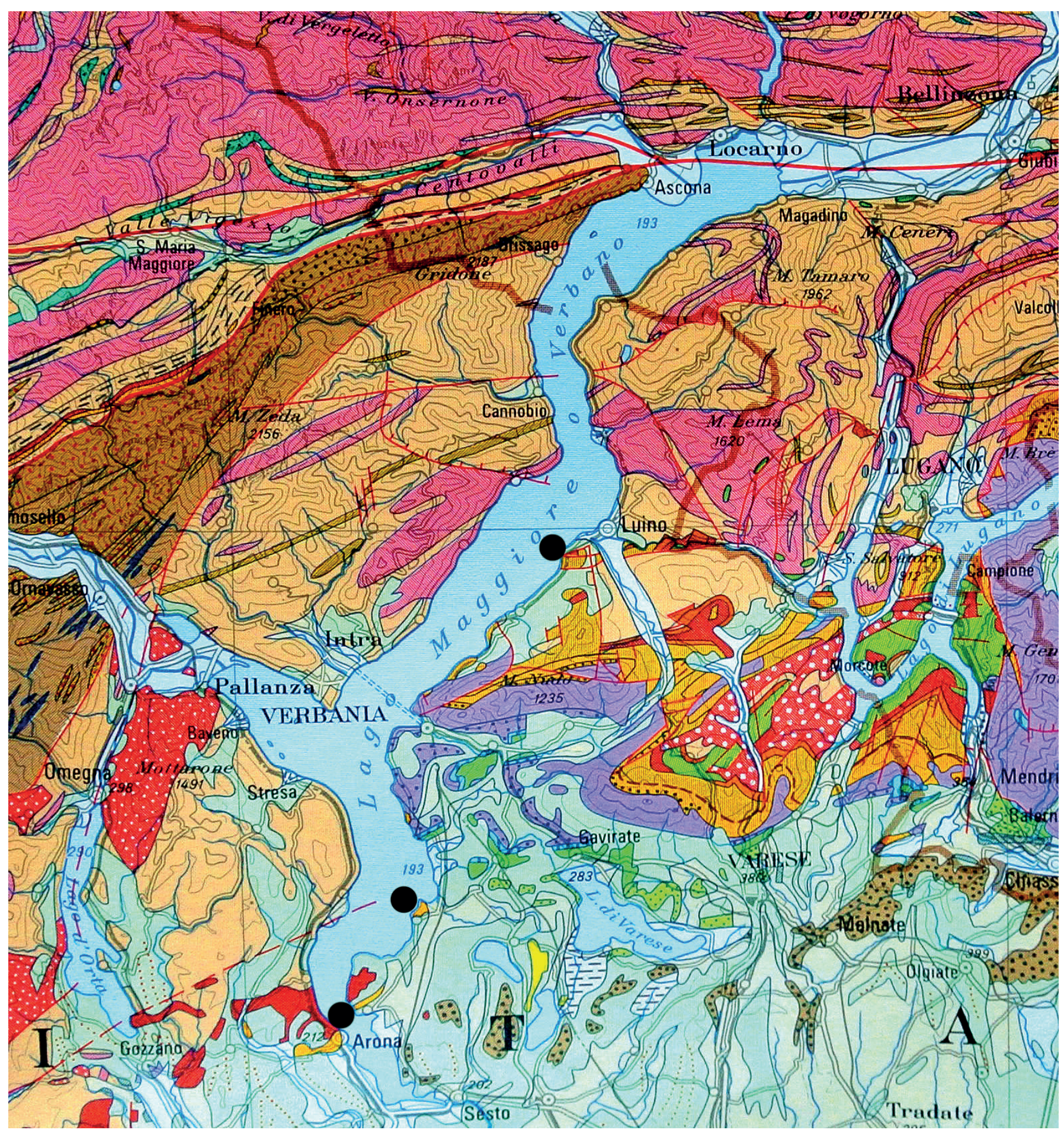

Fig. 2. Gli affioramenti di dolomia (colore arancione) interessano parte della sponda orientale del lago Maggiore. In particolare, sono indicati gli affioramenti di Caldè (in alto), di Ispra (al centro) e di Angera (in basso) (da Geologische Karte der Schweiz 1:500.000, Schweizerischen Geologischen Kommission, Bern 1980)

(Caldè, Ispra) e nei dintorni (Arcisate, Rasa, Brenno Useria), hanno confermato la presenza di ossido di magnesio (intorno al 20\%) insieme con ossido di calcio (intorno al 30\%) (Brusa e Mamoli 1994).

\section{LA CALCE DEL FIUME ADDA}

I corsi dei diversi fiumi che dalle Alpi scendono nella pianura Padana, in particolare quello dell'Adda tra Brivio 


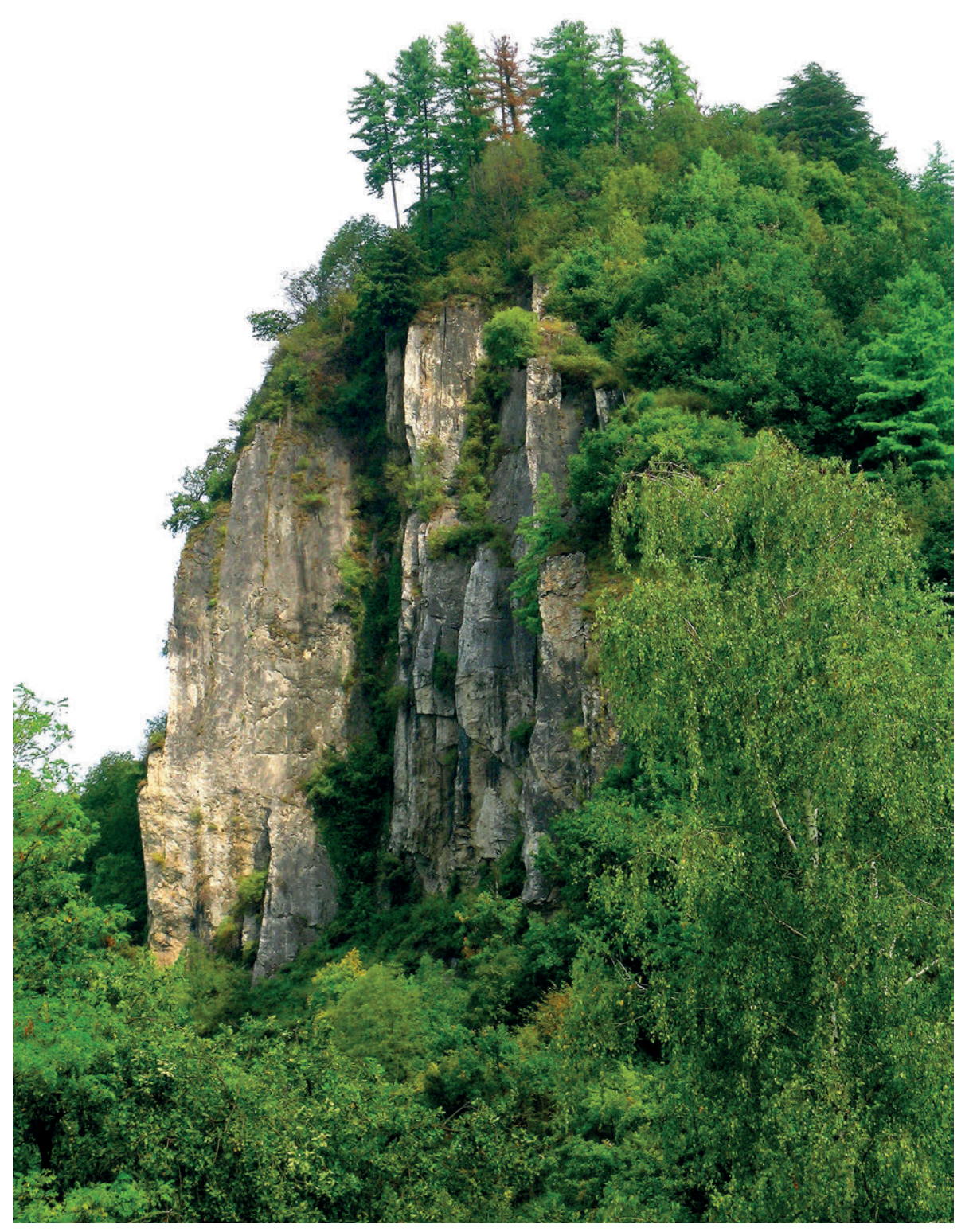

Fig. 3. La Rocca di Caldè (Luino): dolomia disposta in strati verticali e Trezzo, sono interessati da un accumulo di materiale detritico sciolto, riferito al trasporto operato dai ghiacciai quaternari e dai corsi d'acqua stessi (Fig. 6). Si tratta di ciottoli e ghiaie a supporto clastico del Pleistocene - Olocene (Carta Geologica d'Italia; Fig. 7) la cui composizione rispecchia quella del bacino idrografico del fiume Adda: rocce silicatiche di origine metamorfica (gneiss e paragneiss, serpentiniti) e magmatica (graniti, granodioriti e dioriti) nella fascia alpina; rocce sedimentarie di natura carbonatica (Dolomia Principale, Calcare di Èsino) del Triassico e calcari marnosi (Selcifero lombardo) del Giurassico nella fascia prealpina. I ciottoli alimentavano fornaci che producevano la cosiddetta "calce forte" o "calce di Gera d'Adda" (Breislak: cap. 1, parag. 9; Curioni: parte 2, capitolo 5), una calce che presentava caratteri debolmente idraulici per la presenza di una frazione argillosa ed il cui commercio "teneva dietro" a quello della calce magnesiaca. A questo proposito può essere significativo citare il testo di Curioni: “(...) la calce così detta forte, costituita da calcari non dolomitici alquanto argillosi dei terreni liassici, adoperata specialmente per opere murarie esposte all'umido." (Curioni: parte 2, capitolo 5).

Questo tipo di calce non sembra essere stato utilizzato negli intonaci romani finora esaminati: indagini mediante spettrofotometria per fluorescenza ai raggi $\mathrm{X}$ effettuate su alcuni campioni (Università Cattolica, via Correnti) non hanno infatti rilevato la presenza di Silicio nei leganti magnesiaci. 


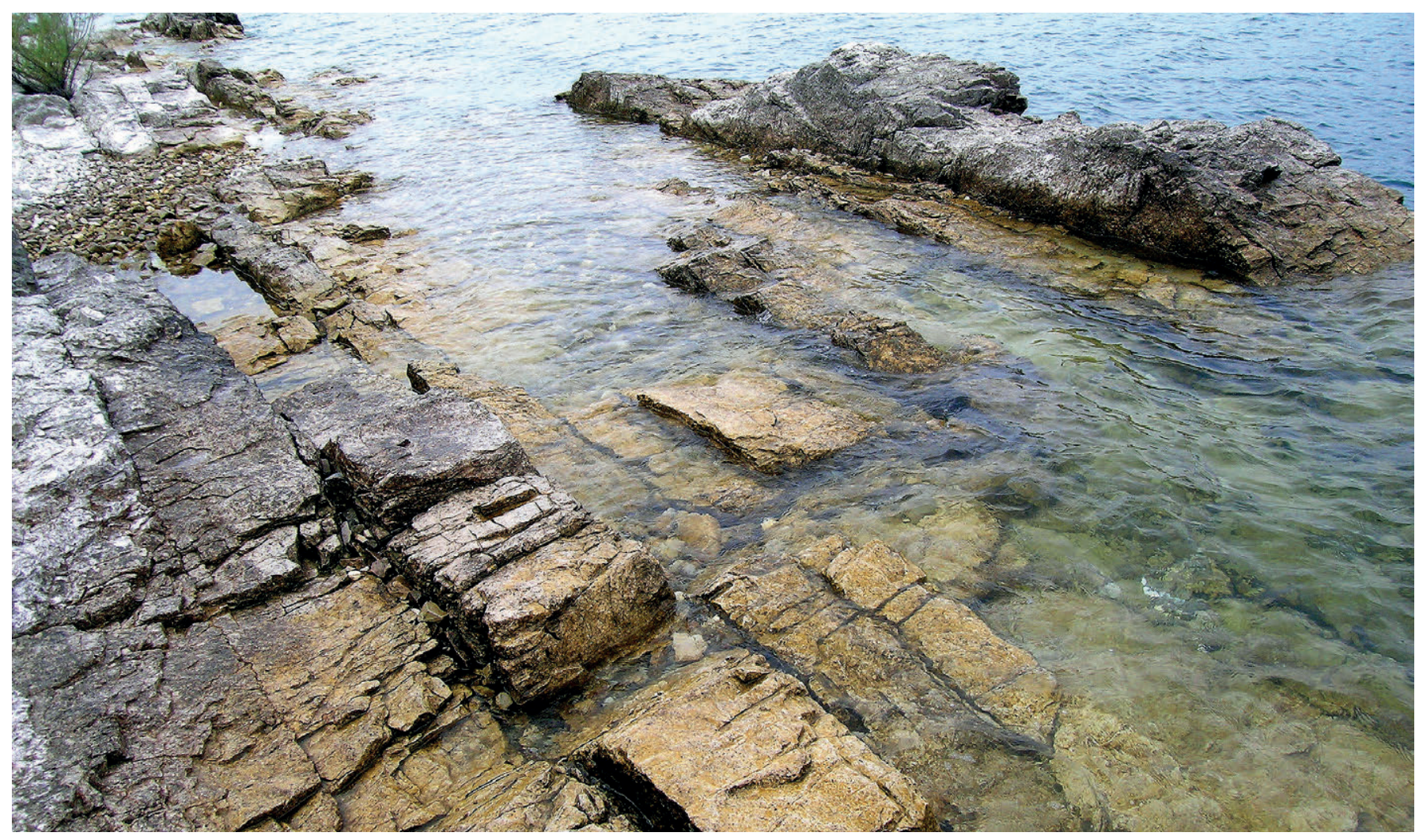

Fig. 4. Gli strati di dolomia a Caldé, sulla riva del lago Maggiore.

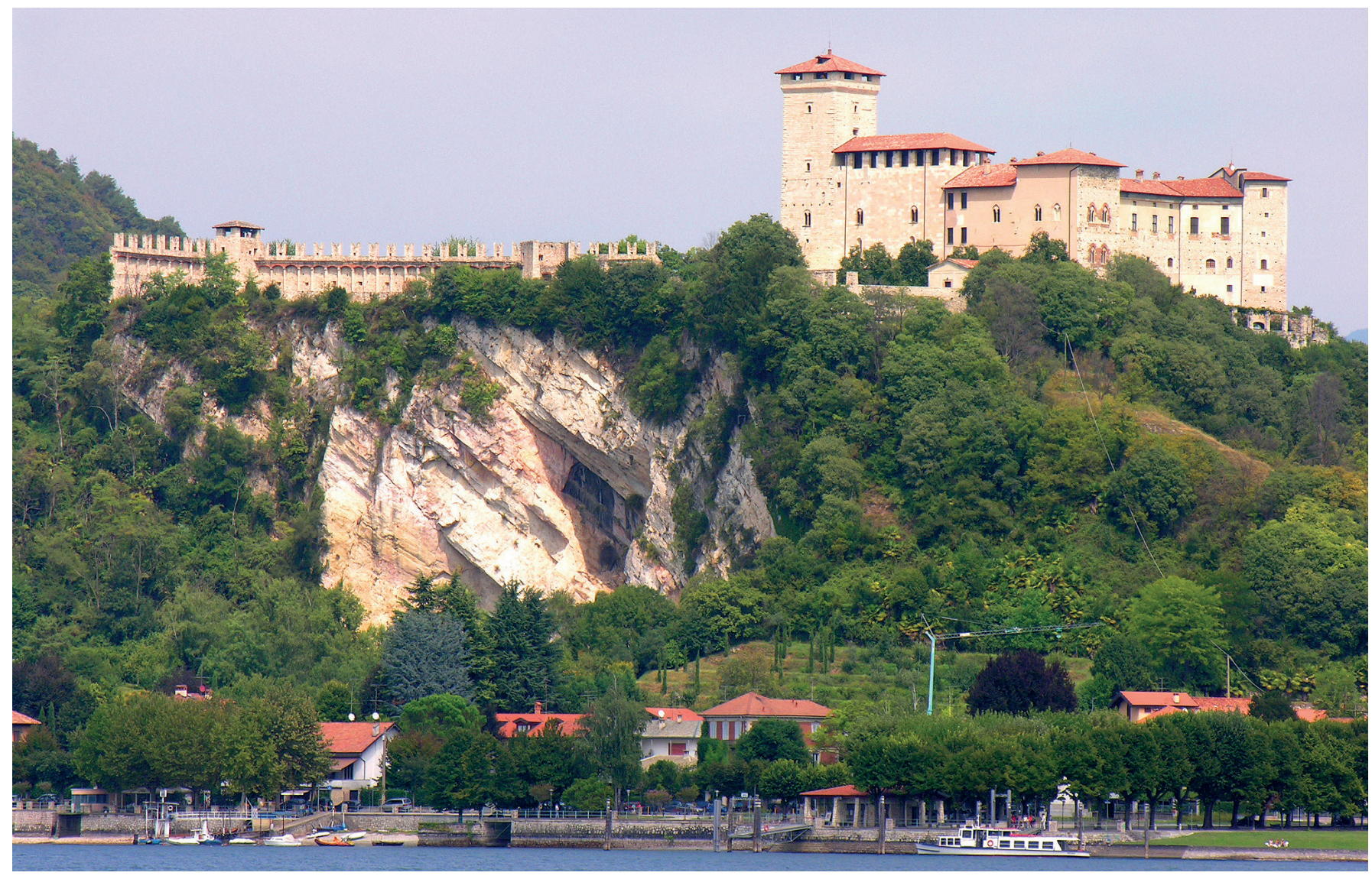

Fig. 5. La cava di dolomia presso Angera e la rocca dei Borromeo. 


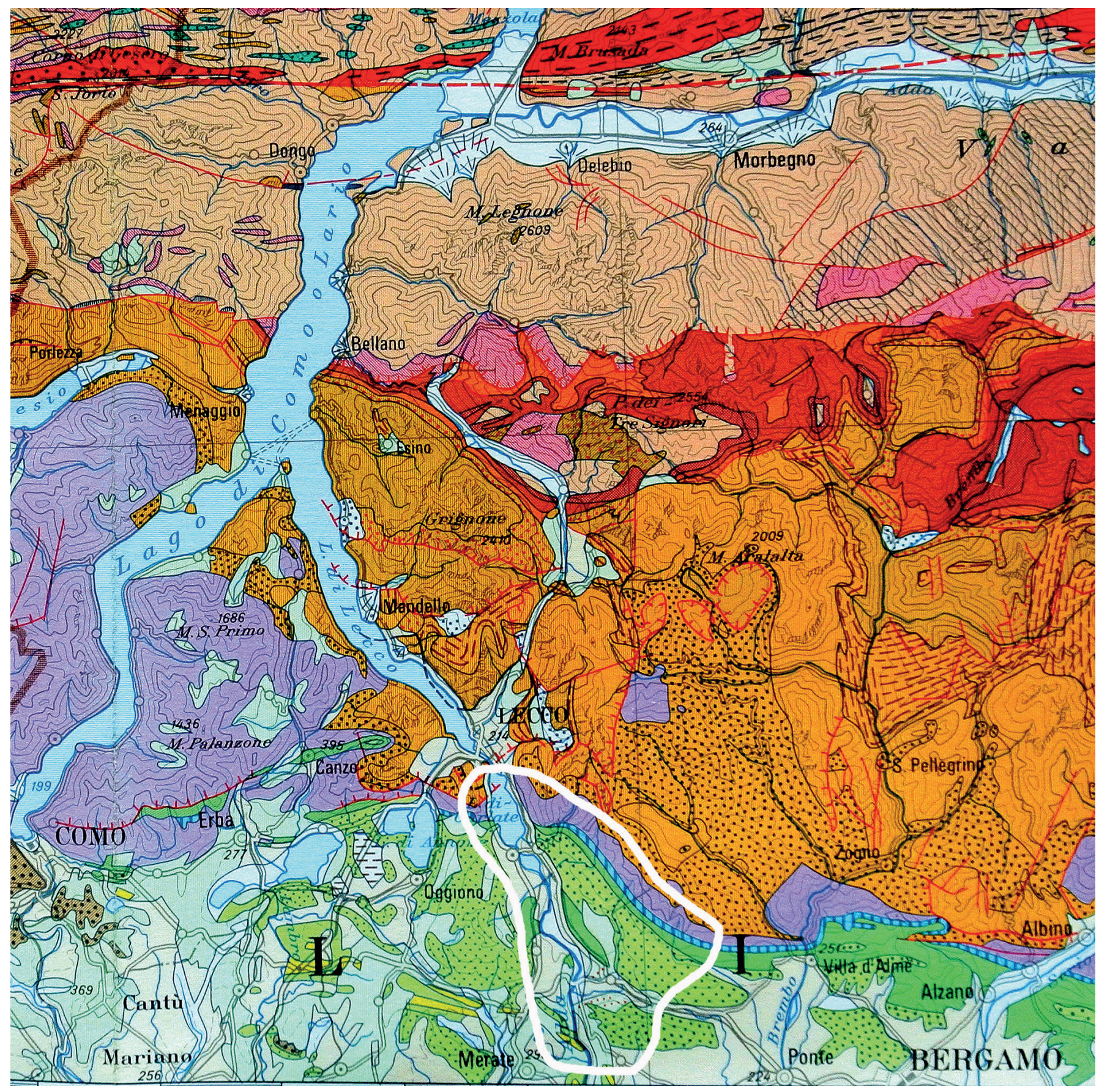

Fig. 6. Il bacino del fiume Adda con affioramenti di dolomie (colore arancione - a Est) e di calcare (colore blu - a Ovest); è indicata anche I'area di affioramento di calcari marnosi (da Geologische Karte der Schweiz).

\section{IL TRASPORTO}

Le cave di pietra da calce del lago Maggiore erano ubicate a brevissima distanza dalla linea di costa, facilitando quindi il caricamento della materia prima su imbarcazioni che, attraverso una fitta rete di vie d'acqua tra Ticino e Po, raggiungevano anche località a grande distanza. Il materiale cavato e cotto sul corso nell'Adda era anch'esso distribuito attraverso le vie d'acqua connesse al corso del fiume stesso.

L'uso, in epoca romana, delle vie d'acqua dell'attuale territorio lombardo (Fig. 8) è testimoniato da Strabone e da Sidonio Apollinare. Strabone, nei primi decenni dopo Cristo, ricorda la navigabilità dei fiumi padani: “(...) e 


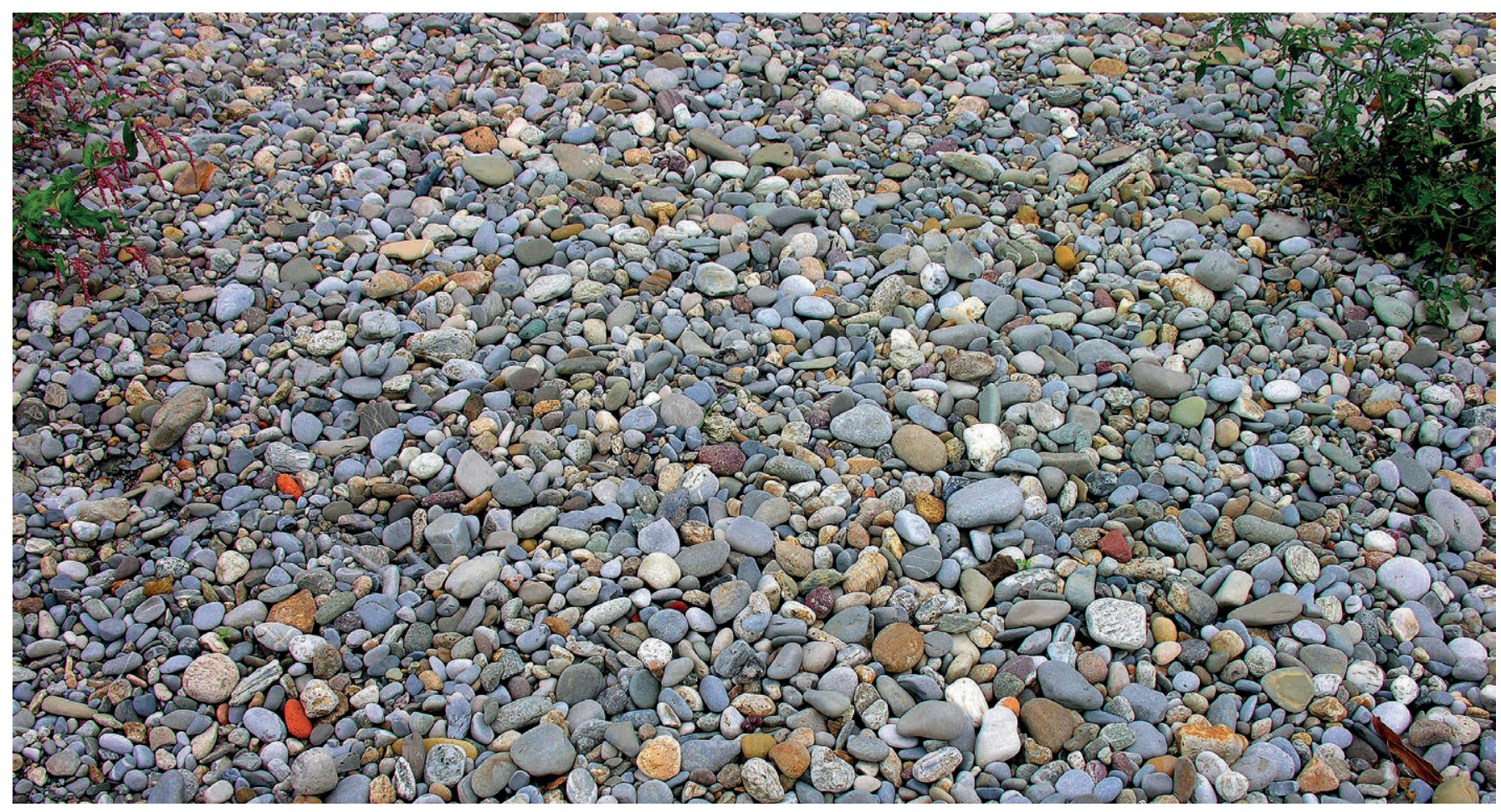

Fig. 7. Deposito sciolto del fiume Adda.

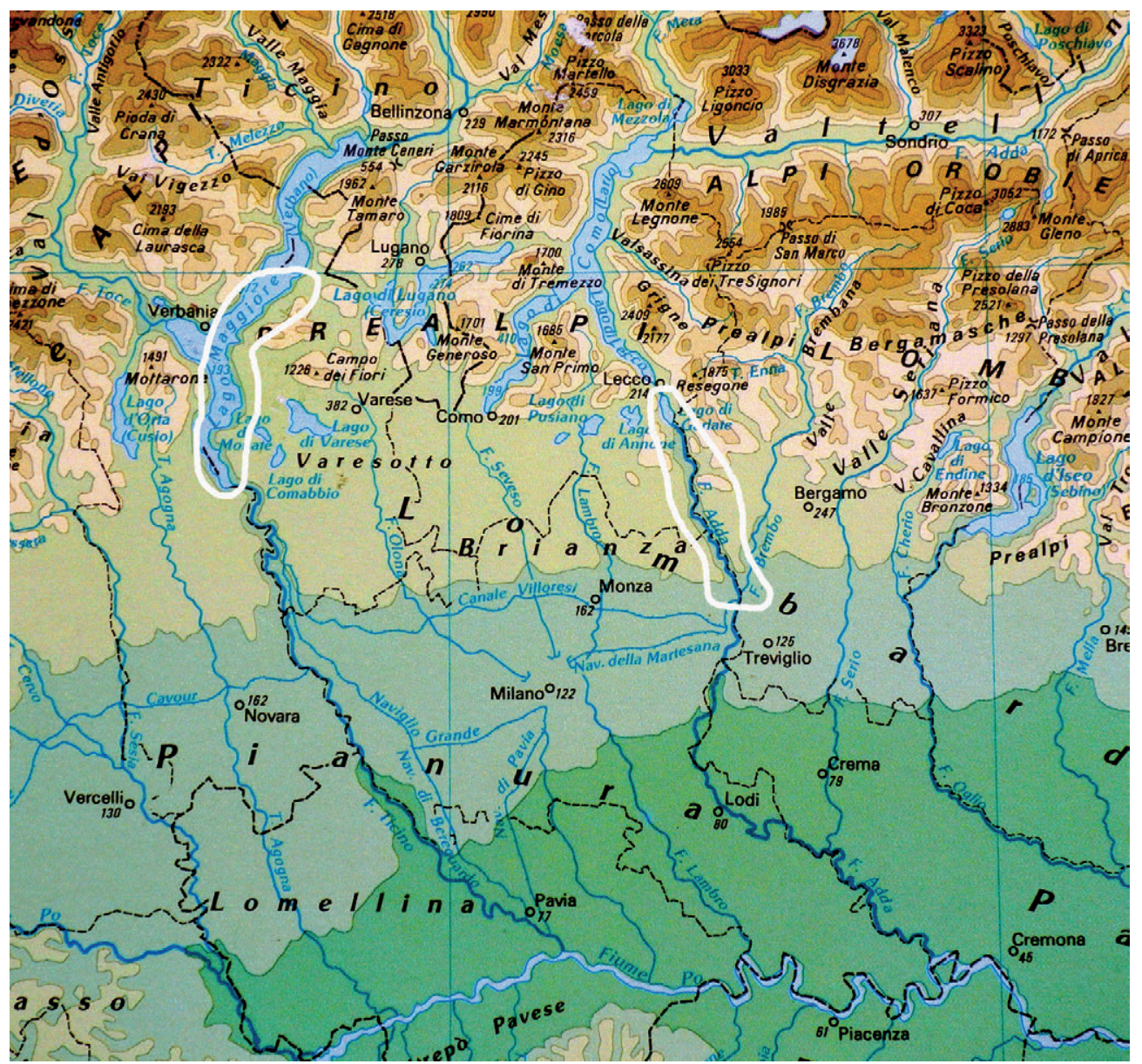

Fig. 8. Carta geografica dell'area lombarda compresa fra i laghi prealpini, Milano e il fiume Po: sono evidenti le vie d'acqua utilizzabili per il trasporto di materiali lapidei e sono indicate le aree di approvvigionamento del Lago Maggiore e del fiume Adda. 
quelle [città] che stanno al di là delle paludi, nella terra ferma, hanno fiumi che si possono risalire fino ad una mirabile distanza. Fra questi è da notare principalmente il Po, il quale è grandissimo per sé medesimo, e spesse volte si gonfia per le piogge e per le nevi." [Strabone: libro $5,1,6]$. Sidonio, alla metà del quinto secolo, ricorda la rapidità degli spostamenti per via fluviale: "A Pavia arrivai a bordo di un battello postale (così chiamano l'imbarcazione) e rapidamente discesi la corrente verso il Po (...)" [Sidonio: libro 1, 5 - A Eronio, 3].

Infine, non potendo considerare eventuali motivi tecnologici che favorissero l'impiego della calce magnesiaca, è possibile ritenere che le dolomie fossero state scelte proprio per la facilità di distribuzione della materia prima ottenuta. Questa ipotesi è suggerita da Curioni: “( ...) concorre a far preferire questa calce [magnesiaca] la facilità del suo trasporto per vie d'acqua, stante che i nostri laghi ne intercettano i potentissimi depositi" (Curioni: parte 2, capitolo 5).

\section{CONCLUSIONI}

La composizione del legante utilizzato in epoca romana per la preparazione degli intonaci, provenienti da diversi siti archeologici milanesi, è stata ritenuta valida per l'identificazione della provenienza delle materie prime e dei luoghi di produzione della calce, in assenza di testimonianze dirette.

La presenza di magnesite (carbonato di magnesio) consente di affermare che la materia prima provenisse unicamente dalla cottura di dolomie (rocce sedimentarie di origine chimica costituite da carbonato doppio di Calcio e Magnesio) e che i relativi siti di cava fossero ubicati nelle formazioni dolomitiche del Triassico affioranti in vari punti della costa orientale del lago Maggiore. Come già verificato a proposito delle cave di pietre da costruzione, si ritiene molto probabile che i siti di cava delle materie prime utilizzate dai Romani per la preparazione della calce siano gli stessi sfruttati poi dal Medioevo fino all'epoca contemporanea.

Tra i motivi dell'uso esclusivo della dolomia, non potendo valutare i criteri tecnologici del tempo, è possibile ritenere che questa pietra fosse stata scelta per la produzione di calce in considerazione del facile collegamento fra gli affioramenti ed il resto della regione, attraverso il lago Maggiore e le vie d'acqua.

\section{BIBLIOGRAFIA}

Alberti, L.B. 1989: De re aedificatoria. Trad. Orlandi, Milano.

Atzeni, C., Massidda, L., Sanna, U. 1996: "Magnesian lime - Experimental contribution to interpreting historical data", Science and Technology for Cultural Heritage, 5 (2), 29-36.

Barbaro, D. 1567: I dieci libri dell'Architettura di M. Vitruvio (...), Venezia. Breislak, S. 1822: Descrizione geologica della provincia di Milano, Milano.

Brusa, M., Mamoli, M. 1994: "Le calci delle Prealpi varesine - cave, flussi commerciali, modi di utilizzo", Politecnico di Milano e Centro CNR "Gino Bozza" - Tesi di laurea in Architettura.

Bugini, R., Folli. L., Lommano, A. 1996: "Plaster technologies in Northern Italy from 12th to 19th century", J. Riederer (ed.), Proceedings 8th International Congress on Deterioration and Conservation of Stone, Berlin, 1495-1504.

Bugini, R., Folli. L. 2013: "Critères pour la comparaison des enduits peints romains de la Lombardie", Archéosciences 37, 41-50.

Bugini, R., Folli, L. 2014: "Le cave romane in Lombardia", J. Bonetto, S. Camporeale, A. Pizzo (eds.), Actas Congreso Arqueología de la construcción IV - Las canteras en el mundo antiguo, Anejos de AEspA LXIX, Mérida, 179-188.

Carta Geologica d'Italia: Foglio 097 Vimercate, ISPRA, Roma.

Cavalieri San Bertolo, N. 1831: Istituzioni di architettura statica e idraulica, Fratelli Negretti, Mantova.

Curioni. G. 1877: Geologia - Parte seconda. Descrizione ragionata delle sostanze estrattive utili, metalliche e terre, raccolte nelle Provincie lombarde, Milano.

Diekamp, A., Konzett, J., Wertl, W., Tessadri. R., Mirwald, P. W. 2008: "Dolomitic lime mortar. A commonly used building material for medieval building in Western Austria and Northern Italy", J.W. Łukaszewicz, P. Niemcewicz (eds.) Proceedings $11^{\text {th }}$ International Congress on Deterioration and Conservation fo Stone, Torun, 597-604.

Fieni, L. 2000: Calci lombarde - Produzione e mercati dal 1641 al 1805, All'Insegna del Giglio, Firenze.

Jagnaux, R. 1885: Traité de minéralogie appliquée aux arts, à l'industrie, au commerce..., Octave Doin éditeur, Paris.

Klemm, W. A. 1989: "Cementitious materials: historical notes", Material Sciences of Concrete, 1, 1-29.

Montagni, C. 1988: "La antiche fornaci liguri”, G. Biscontin, E Vassallo, S. Volpin (eds.) Atti Convegno Scienza e Beni Culturali, Bressanone - Padova, 143a-153a

Mills, A. P. 1915: Materials of Construction, John Wiley, New York.

Palladio, A. 1570: I quattro libri dell'architettura. Venezia.

Palladius 1843: De re rustica. Trad. Cabaret-Dupaty, Paris.

Plinius 1962: Naturalis Historia. Trad. Heichholz, London.

Scamozzi V. 1615: L'idea dell'architettura universale, Venezia.

Schork, J. 2012: "Dolomitic lime in the US - History, Development and Physical Characteristics", Journal of Architectural Conservation, 18, 3, 7-25.

Seeley, N. 2000: "Magnesian and dolomitic lime mortars in bulding conservation", Journal of Architectural Conservation, 6, 2, 21-29.

Sidonio Apollinare 1936: Epistularum libri X. Trad. da Anderson, London.

Strabone 1833: Della geografia. Trad. Ambrosoli, Milano. 